\title{
Integrated Data Analysis for the Quantification of Emotional Responses During Video Observation
}

\author{
Pierluigi Reali, Sergio Cerutti, Anna Maria Bianchi \\ Dept. of Electronic, Informatics, Bioengineering \\ Politecnico di Milano
}

\author{
Debora Bettiga, Lucio Lamberti \\ Dept. of Management, Economics, Industrial Engineering \\ Politecnico di Milano
}

\author{
Alessandra Mazzola, Margherita Pillan \\ Dept. of Design \\ Politecnico di Milano
}

\begin{abstract}
The use of questionnaires at the end of a specific task only evaluates what is expressed by the conscious mind and, therefore, cannot give a complete representation of the individual's emotional state. By adding data from physiological measures, such as cerebral activity, skin conductance, heart rate and gaze position, more accurate information about cognitive engagement and emotional responses to a given task could be provided. This study aims to evaluate participants' emotional (arousal, valence) and cognitive (memorization effort, attention, pleasantness) responses toward two videos, through the integration of above cited measures. Our findings show that the two tested videos produce two different unconscious reactions (one video causes a significantly higher increase in heart rate and the other one requires higher memorization effort), while producing similar conscious responses (no statistically significant differences were found by analyzing questionnaires' answers). Further, eye tracking device provided a way to investigate reasons behind these differences. The results show that the integration of self-reported and biological measures with eye tracking data could effectively help to understand emotional and cognitive responses during video observation.
\end{abstract}

Keywords- electroencephalogram; heart rate variability; skin conductance; eye tracking

\section{INTRODUCTION}

Previous studies have shown that what is expressed by the conscious mind - through interviews, questionnaires, focus groups - is not a complete representation of the individual's emotional state [1]. Collecting data about physiological individual responses, such as cerebral activity, cutaneous impedance and heart rate, and integrating them with data related to individual's gaze position and self-reported measures could provide more interesting and accurate information about cognitive engagement and emotional responses, during the execution of a specific task.

Specifically, this study aims to evaluate participants' emotional and cognitive responses toward two videos, characterized by a common main theme, by employing physiological, self-reported and eye-tracking measures. Both the videos were presented in two versions: static, that does not allow any user interaction, and interactive, that allows the user to interact with the video through buttons displaying additional content, activating music etc.

The laboratory experiments were conducted at PHEEL (Physiology Emotion Experience Laboratory), the biomarketing laboratory of Politecnico di Milano, Italy.

\section{MEthodS}

\section{A. Experimental Design}

Fourteen healthy volunteers ( 7 males and 7 females) were recruited for the study. The sample size is in line with prior experiments adopting biometric measures [2][5]. All volunteers were welcomed and briefly explained what the experimental protocol would have consisted of and were told they could withdraw from the experiment at any time. Subjects were instructed that after video watching they will be asked to complete a questionnaire to record their evaluation. This procedure, according to prior research [3], serves to prime respondents to engage into stimuli evaluation. Informed consent was obtained from each of them before the experiment began; after that, participants were asked to comfortably sit on a reclining chair, while all the necessary sensors were being placed on their skin. During the experiment, subjects were seated in front of a PC monitor used for stimuli delivery. To assure the absence of any kind of social influence or disturbance, the experiment was performed on one subject at a time. Particular attention was paid during each phase in order to make the experience as pleasant as possible and to avoid possible variations of participants' anxious state during the experiment.

Each subject was asked to watch two videos, one static and the other one interactive. The static one had an approximate duration of 15 seconds, the interactive one had similar duration but provided the possibility to look for additional content. Subjects were instructed to freely explore the website page and to click on the "play" button whenever they like, making the video clip to start. A neutral image was shown for two minutes before the first video, in order to make participants relaxing and create a baseline reference for each acquired signal. At the end of the second video clip, all the sensors were removed and 


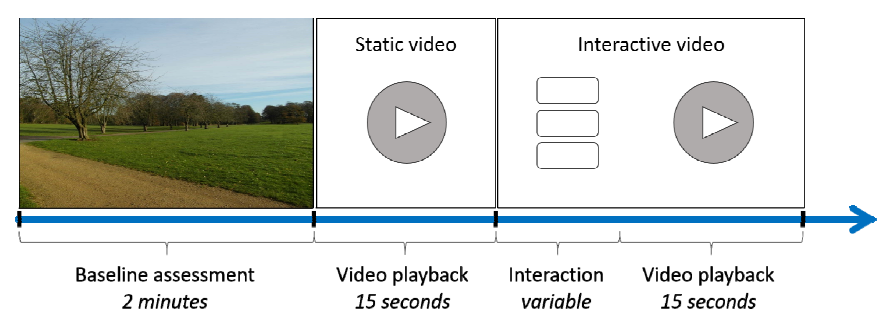

Fig. 1. Time course of the experimental protocol.

participants were taken to another room and asked to complete a questionnaire. A timeline of the experiment is shown in Fig. 1.

By measuring the difference between activity and baseline states instead of absolute quantities, it is possible to compare responses obtained by different subjects to the same stimuli [4]. This aspect makes the acquired responses unrelated to the initial state of each participant, consequently reducing intersubjects' variability.

\section{B. Videos for stimulation}

Two different videos were evaluated, both administered in a static and an interactive version. The videos were characterized by a common main theme and were presented to participants in random order to avoid sequence-related effects on study's results. All the video sequences which had been considered in the study are reported in Tab.1.

TABLE I. APPLIED VIDEO SEQUENCES

\begin{tabular}{|l|l|l|}
\hline \multicolumn{1}{|c|}{$\mathbf{1}^{\circ}$ video } & \multicolumn{1}{|c|}{$\mathbf{2}^{\circ}$ video } & Num. of Subjects \\
\hline V1 Static & V2 Interactive & 4 \\
\hline V2 Static & V1 Interactive & 4 \\
\hline V1 Interactive & V2 Static & 3 \\
\hline V2 Interactive & V1 Static & 3 \\
\hline
\end{tabular}

\section{Biological Measures}

In the present study, the following biological signals were recorded and analyzed.

Electroencephalography (EEG) was acquired by means of a portable 64-channels system (SD LTM Express and System Plus Evolution software, Micromed, Italy). To facilitate and speed-up electrodes positioning, a 64 electrodes pre-cabled cap was used. In total, 27 electrodes uniformly distributed on the scalp were used and each electrode was filled with a waterbased gel to enhance conductivity between each electrode and participants' scalp. The EEG activity was collected at a sampling rate of $256 \mathrm{~Hz}$ and the impedance level was kept below $5 \mathrm{k} \Omega$. EEG traces were automatically segmented to separate EEG activity recorded during the baseline period from the one acquired during the observation of the videos. The preprocessing done on recorded EEG comprised band pass filtering (high pass $=1 \mathrm{~Hz}$; low pass $=35 \mathrm{~Hz}$ ) and the execution of Independent Component Analysis (ICA) to detect and remove components related to eye and muscular artifacts. The original EEG signal was referred to a specific electrode placed on the pre-cabled cap, between $\mathrm{CPz}$ and $\mathrm{Pz}$; this signal has been transformed by means of the Common Average Reference (CAR), in order to calculate Memorization Index (MI), Attention Index (AI) and Pleasantness Index (PI) as suggested in the literature [2] [5]. Individual Alpha Frequency (IAF) for each subject was calculated [6] and, then, EEG traces were band pass filtered to obtain three different signals, each one containing different contributions to the total EEG spectrum. The three frequency bands were chosen according to the literature [2] and, specifically, they were:

- Theta, which ranges between IAF-6Hz and IAF-4Hz;

- Lower alpha, between IAF-4Hz and IAF;

- Upper alpha, between IAF and IAF+2Hz.

Theta band pass filtered channels AF3, F3 were used for the calculation of MI; lower alpha frequency band was considered for channels Fpz, AF3, F3, AF4, F4, Fz to compute AI; finally, upper alpha band pass filtered channels F3, AF3, F4, AF4 were used to compute PI [2]. These three indices are obtained by computing Global Field Power (GFP) [7] between listed signals.

The average of each index was calculated during baseline acquisition and subtracted to the mean value computed during the videos. All the values obtained in this way were standardized from 0 (minimum value obtained from all the subjects) to 1 (maximum) [8], with the aim of making readily understandable which one of the observed videos caught more attention (AI), required greater memorization efforts (MI) or showed to be more pleasant for the spectators (PI).

Electrocardiography (ECG) and Skin Conductance (SC) were both acquired through ProComp Infiniti system and Biograph Infiniti software (Thought Technology Ltd., Canada). The first signal was recorded at a sampling rate of $2048 \mathrm{~Hz}$, which is more than enough to obtain a reasonably accurate representation of QRS complexes [9], while the second one was collected with a sampling rate of $256 \mathrm{~Hz}$. By analyzing these two signals it is possible to monitor autonomic nervous system activity and assess the emotional state of the participants [10]. In particular, heart rate variability (HRV) signal (temporal distance between consecutive $\mathrm{R}$ peaks) was extracted from ECG through recognition of $\mathrm{R}$ peaks, which was performed by means of Pan-Tompkins' method [11]; heart rate (HR) was then calculated by computing the reciprocal of each R-R interval. Mean HR and SC were evaluated, separately, during baseline and videos and, in order to obtain the average percent increase of HR and SC during video watching, the following ratio (1) was applied to both measures:

$$
\text { Mean Perc.Incr. }=100 \cdot\left(\mu_{\text {video }}-\mu_{\text {baseline }}\right) / \mu_{\text {baseline }}
$$

where $\mu_{\text {baseline }}$ and $\mu_{\text {video }}$ stand for average value of HR and SC during baseline acquisition and video observation, respectively.

Frequency-domain (i.e. based on the estimation of power spectral density (PSD)) measures were not considered, due to videos' short duration. In fact, the number of heartbeats occurred during each video playback is not large enough for accurate PSD computing [9]. 


\section{Eye tracking}

A wearable eye tracking device (SensoMotoric Instruments Eye Tracking Glasses, Germany) was used to obtain participants' gaze position during video watching. Eye tracking devices provide information about visual exploration strategies, mental workflows, and cognitive processes [12] [13].

We explored the scanpath (the two-dimensional movement composed of alternating saccades and fixations [14]) of each subject, by analysing saccades and fixations during the subject visual exploration. Saccades are fast movements of the eyes that rapidly shift the gaze from one position to another. Fixation is the time between two successive saccades and it represents the period of time during which visual information can be acquired. We quantitatively analyzed the path of eye movements in terms of:

- identification and analysis of specific areas (number of fixations, average time, glance time);

- exploration sequence.

\section{E. Self-reported Measures}

Self-reported emotional responses of arousal, pleasure, and involvement were collected through the mean of a questionnaire, using validated scales after video watching. According to prior research ([15], [16]) we used the scale developed by Mehrabian \& Russell (1974) [17] to measure arousal-quietness dichotomy. The arousal scale items used, listed in random order, were "excited-calm", "stimulatedrelaxed", "aroused-unaroused", "sluggish-frenzied", "dulljittery" and "sleepy-wide awake". Also pleasure was measured with Mehrabian \& Russell (1974) scale. The specific questions for pleasure were five semantic differential items, randomly presented: "happy-unhappy," "pleased-annoyed", "satisfiedunsatisfied, "melancholic-contented", "despairing-hopeful" and "bored-relaxed".

\section{RESULTS}

In the present paragraph will be illustrated the results obtained in the experiment described above.

\section{A. Self-reported Measures}

Tab. 2 and Tab. 3 show descriptive statistics about selfreported data of arousal, pleasure and involvement.

To detect if the stimuli generated significantly different emotional and cognitive reactions in participants, we performed One-Way Analysis of Variance tests. In a first analysis, we explored if the static and the interactive video generated different reactions. Results show that there are no significant differences in the arousal, pleasure and involvement between static and interactive video versions. A further analysis explored if differences exists between the two videos (V1 and V2), resulting in no significant differences in terms of participants' emotional reactions.
TABLE II. SELF-REPORTED STATISTICS PER VIDEO CONTENT

\begin{tabular}{|c|c|l|l|l|}
\hline \multicolumn{2}{|c|}{ VIDEO CONTENT } & involvement & arousal & pleasure \\
\hline \multirow{2}{*}{ V1 } & Mean & 5.00 & 4.57 & 4.42 \\
\cline { 2 - 5 } & St.dev. & 1.32 & 1.14 & 0.653 \\
\hline \multirow{2}{*}{ V2 } & Mean & 5.29 & 4.15 & 4.82 \\
\cline { 2 - 5 } & St.dev & 0.932 & 1.15 & 0.681 \\
\hline
\end{tabular}

TABLE III. SELF-REPORTED STATISTICS PER VIDEO TYPOLOGY

\begin{tabular}{|c|c|l|l|l|}
\hline \multicolumn{2}{|c|}{ VIDEO TYPOLOGY } & involvement & arousal & pleasure \\
\hline \multirow{2}{*}{ Static } & Mean & 5.40 & 4.43 & 4.74 \\
\cline { 2 - 5 } & St.dev. & 0.962 & 1.07 & 0.653 \\
\hline \multirow{2}{*}{ Interactive } & Mean & 4.88 & 4.30 & 4.50 \\
\cline { 2 - 5 } & St.dev & 1.26 & 1.25 & 0.722 \\
\hline
\end{tabular}

\section{B. Biological Measures}

Fig.2 and Fig.3 summarize for all the participants the results obtained through the analysis of EEG, ECG and SC signals.

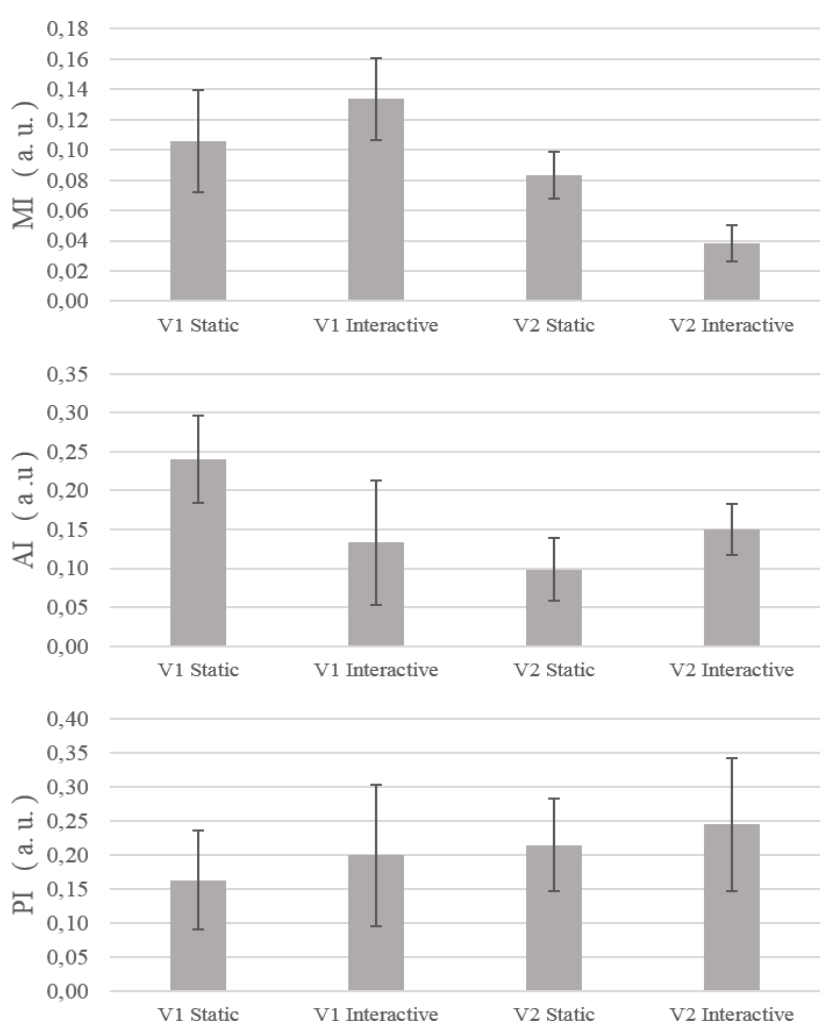

Fig. 2. Average value and mean standard error of Memorization Index (MI), Attention Index (AI) and Pleasantness Index (PI), reported for each showed video. 

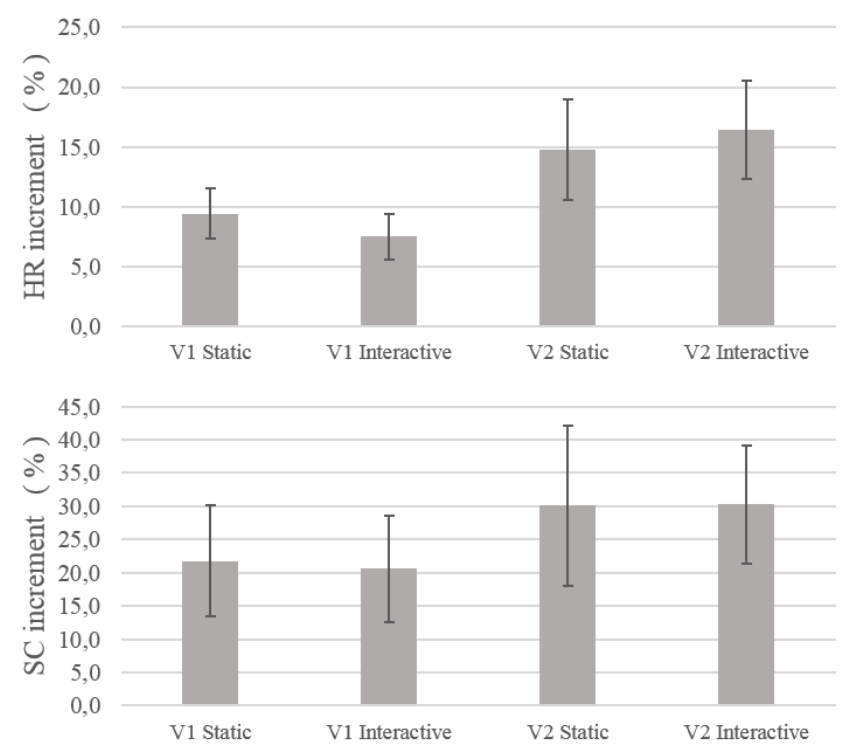

Fig. 3. Average value and mean standard error of the percent increase of HR and SC, during the observation of each video.

EEG related variables (baseline-subtracted and standardized values of MI, AI and PI) provide information about central nervous system activity, giving a measure of the cognitive engagement required by the administered task, while the percent increase of SC and HR during video observation provide details about autonomic nervous system activation, quantifying participants' emotional state in terms of arousal and valence, respectively [4]. Specifically, an increment of SC during video watching reflects sympathetic arousal increase, while an increment of HR is mediated by para-sympathetic balance and reflects more positive valence (i.e. pleasant attitude toward the video) [18] [19].

To analyze statistically significant differences between the four considered groups (i.e. V1 Static, V1 Interactive, V2 Static, V2 Interactive) One-Way Analysis of Variance tests were performed. No statistically significant differences emerged from the analysis but, as could be seen from Fig.2, AI seems to have a growing pattern from the first video to the last one, while PI shows a decreasing pattern moving in the same direction. From Fig.3 a general increase of HR and SC emerges during video watching compared to the baseline, which may indicate that, in general, subjects have found all the videos pleasant and somewhat engaging.

Tab. 4 and Tab. 5 show descriptive statistics about biological measures, re-organized by video content and typology.

To detect statistically significant differences between the two considered video clips, One-Way Analysis of Variance tests were performed. First, we explored possible differences between static and interactive videos. Results show that there are no significant differences for cognitive nor for emotional biological variables.
TABLE IV. Biological Measures Statistics Per Video CONTENT

\begin{tabular}{|c|c|l|l|l|l|l|}
\hline \multicolumn{2}{|c|}{ VIDEO CONTENT } & \multicolumn{1}{|c|}{ HR } & SC & MI & AI & PI \\
\hline \multirow{2}{*}{ V1 } & Mean & $8.47^{\mathrm{a}}$ & 21.18 & $0.121^{\mathrm{a}}$ & 0.182 & 0.180 \\
\cline { 2 - 7 } & St.dev. & 5.23 & 20.83 & 0.072 & 0.172 & 0.205 \\
\hline \multirow{2}{*}{ V2 } & Mean & 15.63 & 30.20 & 0.063 & 0.122 & 0.231 \\
\cline { 2 - 7 } & St.dev & 10.59 & 26.89 & 0.040 & 0.090 & 0.200 \\
\hline
\end{tabular}

TABle V. Biological Measures Statistics Per Video Tipology

\begin{tabular}{|c|c|l|c|c|c|c|}
\hline \multicolumn{2}{|c|}{ VIDEO TYPOLOGY } & \multicolumn{1}{|c|}{ HR } & SC & MI & AI & PI \\
\hline \multirow{3}{*}{ Static } & Mean & 12.12 & 25.94 & 0.094 & 0.163 & 0.187 \\
\cline { 2 - 7 } & St.dev. & 8.90 & 26.61 & 0.060 & 0.133 & 0.166 \\
\hline \multirow{3}{*}{ Dynamic } & Mean & 11.98 & 25.44 & 0.090 & 0.141 & 0.224 \\
\cline { 2 - 7 } & St.dev & 9.37 & 22.20 & 0.071 & 0.148 & 0.234 \\
\hline
\end{tabular}

A further analysis explored if differences exist between the two video contents (V1 and V2). HR and MI show statistically significant differences between the two videos. In detail, percent increase of HR was higher during V2 observation and MI value was higher during V1 observation.

\section{Eye tracking}

Participants' gaze analysis brought to the following considerations.

- For all the participants, the exploration concentrates in the middle part of the screen, where the animation is played.

- All the participants showed low attention at the interactive areas and low tendency to click the control keys.

- Dynamic version of video V1 are ambiguous and the observed subjects did not understand its functionalities (e.g. they don't get that the on/off icon activate and disable audio).

\section{DISCUSSION}

In the present context of analysis, self-reported measures and biological variables seem to lead to two different conclusions. In fact, while Memorization Index and Heart Rate suggest statistically significant differences between the two videos content in terms of cognitive engagement and emotional reaction, participants' answers to the questionnaire indicate that the two videos produced similar involvement, arousal and pleasure sensation. These different results seem to suggest that, for the two videos, conscious evaluation after video observation (assessed through self-reported measures) and unconscious emotional reactions (revealed through biological measurements) do not match. Disagreement between these two kinds of measure has already been reported in the recent literature and could be due to people's incapacity or unwillingness to fully explain their preferences through questionnaires or interviews [20] [21] [22]. 
Specifically, increased Heart Rate reveals that video V2 is perceived to be more pleasant than the other one [18], while Memorization Index shows higher memorization effort during the observation of video V1. These results are in line with prior researches, which show that people could also be interested in unpleasant things [23] because attention is attracted by stimuli that are motivationally relevant, independently of their judged valence [24]; also the qualitative relation between Pleasantness Index and Attention Index highlighted in the previous section (the first index increasing while the second one decreasing through the four tested groups) supports this consideration.

Eye tracking evaluation integrates self-reported and biological results, showing that participants' concentration was more attracted by the two video clips than by the interaction opportunities offered through interactive videos. This result seems to be confirmed by the absence of statistically significant differences between the two video typologies, both for biological variables and self-reported measures.

However, the quite small sample size considered in the present study brings to high variability in the biological measures. Therefore, further studies with an increased sample size are required in order to achieve less measure variability and validate our findings.

\section{ACKNOWLEDGMENT}

We would like to thank PHEEL organization for providing the instrumentation and the spaces needed for the study. P. R. also thanks LINK project (H2020 Grant Agreement No. 692023) for the given support.

\section{REFERENCES}

[1] M.D. Robinson and G.L. Clore, "Belief and feeling: evidence for an accessibility model of emotional self-report", Psychologycal Bullettin, vol. 128, No. 6, 2002, pp. 934-960.

[2] G. Vecchiato, P. Cherubino, A.G. Maglione, W. Kong, S. Hu, D. Wei, A. Colosimo, F. Babiloni, "Comparison of cognitive and emotional cerebral variables in Eastern subjects watching TV advertisements: a case study", International Journal of Bioelectromagnetism, vol.14, No.3, 2012, pp. $127-132$.

[3] D. Kempf and R. Smith, "Consumer processing of product trial and the influence of prior advertising: A structural modeling approach", Journal of Marketing Research, vol.35, No.3, 1998, pp.325-338.

[4] M. Chaouachi, C. Frasson (2010), "Exploring the relationship between learner EEG mental engagement and affect". In: V. Aleven, J. Kay, J. Mostow (eds) Intelligent Tutoring Systems. ITS 2010. Lecture Notes in Computer Science, vol. 6095. Springer, Berlin, Heidelberg.

[5] G. Vecchiato, L. Astolfi, F.D.V. Fallani, F. Cincotti, D. Mattia, S. Salinari, R. Soranzo, F. Babiloni, "Changes in Brain Activity During the Observation of TV Commercials by Using EEG, GSR and HR Measurements", Brain Topogr., vol.23, 2010, pp.165-179.

[6] W. Klimesch, "EEG alpha and theta oscillations reflect cognitive and memory performance: a review and analysis", Brain Res. Rev., vol.29, No.2, 1999, pp. 169-195.
[7] D. Lehmann and W. Skrandies, "Reference-free identification of components of checkerboard-evoked multichannel potential fields", Electroencephalogr. Clin. Neurophysiol., vol.48, 1980, pp. 609-621.

[8] C. Vercellis, "Business intelligence: modelli matematici e sistemi per le decisioni”, Mc Graw Hill Education, 2006, p.97.

[9] Task Force of The European Society of Cardiology and The North American Society of Pacing and Electrophysiology, "Heart rate variability: standards of measurement, physiological interpretation, and clinical use", European Heart Journal, vol.17, 1996, pp.354-381.

[10] N. Montano, A. Porta, C. Cogliati, G. Costantino, E. Tobaldini, K.R. Casali, F. Iellamo, "Heart rate variability explored in the frequency domain: a tool to investigate the link between heart and behavior", Neurosci. Biobehav. Rev., vol.33, No.2, 2009, pp.71-80.

[11] J. Pan, W.J. Tompkins, "A real-time QRS detection algorithm", IEEE Transactions on Biomedical Engineering, vol. BME-32, No.3, pp.230236, March 1985.

[12] M. Andrzejewska, A. Stolińska, "Comparing the Difficulty of Tasks Using Eye Tracking Combined with Subjective and Behavioural Criteria", Journal of Eye Movement Research, vol.9, No.3, 2016, pp.116.

[13] E. Carniglia, M. Caputi, V. Manfredi, D. Zambarbieri, E. Pessa, "The influence of emotional picture thematic content on exploratory eye movements", Journal of Eye Movement Research, vol.5, No.4, 2012, pp.1-9.

[14] S.P. Liversedge, I. Gilchrist, S. Everling, "The Oxford Handbook of Eye Movements", Oxford University Press, 2011.

[15] D. Kempf, "Attitude formation from product trial: Distinct roles of cognition and affect for hedonic and functional products", Psychology \& Marketing, vol.16, No.1, 1999, pp.35-50.

[16] W. Havlena, M. Holbrook, "The varieties of consumption experience: comparing two typologies of emotion in consumer behavior", Journal of consumer research, vol.13, No.3, 1986, pp.394- 404.

[17] A. Mehrabian, J. Russell, "An approach to environmental psychology”, Cambridge, MA: The MIT press, 1974.

[18] A.C. Granero, F. Fuentes-Hortado, V.N. Ornedo, J.G. Provinciale, J.M. Ausìn, M.A. Raya, "A comparison of physiological signal analysis techniques and classifiers for automatic emotional evaluation of audiovisual contents", Frontiers in Computational Neuroscience, vol.10, July 2016, Article 74.

[19] M. Bensafi, C. Rouby, V. Farget, B. Bertrand, M. Vigouroux, A. Holley, "Autonomic nervous system responses to odours: the role of pleasantness and arousal”, Chem. Senses, vol.27, 2002, pp.703-709.

[20] G. Vecchiato, L. Astolfi, F. De Vico Fallani, J. Toppi, F. Aloise, F. Bez, D. Wei, W. Kong, J. Dai, F. Cincotti, D. Mattia, F. Babiloni, "On the use of EEG or MEG brain imaging tools in neuromarketing research", Computational Intelligence and Neuroscience, vol.2011, Article ID 643489.

[21] G. Vecchiato, G. Flumeri, A.G. Maglione, P. Cherubino, W. Kong, A. Trettel, F. Babiloni, "An electroencephalographic peak density function to detect memorization during the observation of TV commercials". Conf. Proc. IEEE Eng. Med. Biol. Soc., 2014, pp.6969-6972.

[22] V.C.R. Fortunato, J. de M.E. Giraldi, J.H.C. de Oliveira, "A review of studies on neuromarketing: practical results, techniques, contributions and limitations", Journal of Management Research, vol.6, No.2, 2014, pp.201-220.

[23] S.A. Turner Jr. and P.J. Silvia, "Must interesting things be pleasant? A test of competing appraisal structures", Emotion, vol.6, No.4, 2006, pp.670-674.

[24] M.M. Bradley, "Emotion and motivation", in J.T. Cacioppo, L.G. Tassinary, G.G. Berntson, Handbook of Psychophysiology. Cambridge University Press, New York, 2000, pp.602-642. 